\title{
IrMn as exchange-biasing material in systems with perpendicular magnetic anisotropy
}

\author{
Sebastiaan van Dijken, ${ }^{\text {a) }}$ Magali Besnier, Jerome Moritz, and J. M. D. Coey \\ SFI Trinity Nanoscience Laboratory, Physics Department, Trinity College, Dublin 2, Ireland
}

(Presented on 8 November 2004; published online 13 May 2005)

\begin{abstract}
Contact between a $\mathrm{Co} / \mathrm{Pt}$ multilayer and an IrMn film leads to perpendicular exchange bias. The exchange bias field does not depend on the degree of (111) film texture and for $\mathrm{Co} / \mathrm{Pt}$ multilayers with IrMn at the bottom it can be enhanced by magnetic field annealing. The perpendicular exchange bias of the $\mathrm{Co} / \mathrm{Pt}-\mathrm{IrMn}$ system is limited by a misalignment between the Co spins and the film normal, which is due to a negative magnetic anisotropy contribution from the $\mathrm{Co} / \mathrm{IrMn}$ interface $\left(K_{S}^{\mathrm{Co} / \mathrm{IrMn}}=-0.09 \mathrm{~mJ} / \mathrm{m}^{2}\right)$. The insertion of a $3 \AA$ thick Pt layer at the Co/IrMn interface maximizes the perpendicular exchange-bias field. (C) 2005 American Institute of Physics.
\end{abstract}

[DOI: $10.1063 / 1.1855699$ ]

Exchange-biased ferromagnetic (FM) films with in-plane magnetization are utilized in applications such as read heads, magnetic sensors, and magnetic random access memory. Exchange bias in these structures is established by contacting the FM film with an antiferromagnetic (AFM) layer and the effect manifests itself by a shift in the hysteresis loop and enhanced coercivity. ${ }^{1}$ For practical applications, IrMn is an interesting AFM material since it combines good corrosion resistance with relatively high interfacial exchange energy, high blocking temperature $\left(T_{B} \approx 250{ }^{\circ} \mathrm{C}\right)$, and small critical thickness. ${ }^{2-7}$ Although it has been intensively studied for systems with in-plane magnetization, it has hardly been used in recent studies on perpendicular exchange bias. ${ }^{8-18}$ In a recent paper, Sort et al. showed that Co/Pt multilayers with out-ofplane magnetization can be biased by growing an IrMn layer on top. ${ }^{19}$ In this paper we present an overview on the exchange-biasing properties of IrMn in systems with perpendicular magnetic anisotropy. We show that $\mathrm{Co} / \mathrm{Pt}$ multilayers with an IrMn film on top or at the bottom exhibit a considerable perpendicular exchange bias in the as-deposited state. Magnetic field annealing (bottom-pinned structures) and/or the insertion of a thin Pt layer at the Co/IrMn interface further enhances the exchange-bias field.

The Co/Pt multilayers were deposited by dc magnetron sputtering onto thermally oxidized $\mathrm{Si}$ substrates. A metal shadow mask was used to define Hall bars with a linewidth of $200 \mu \mathrm{m}$. The relatively large extraordinary Hall effect (EHE) of the Co/Pt-IrMn structures, which depends linearly on the out-of-plane moment, was utilized to measure the exchange bias field, the coercivity, and the perpendicular magnetic anisotropy. The crystalline structure of the multilayers was characterized with $\mathrm{x}$-ray diffraction (XRD). For the field annealing experiments we used a vacuum furnace $(p<1$ $\times 10^{-6} \mathrm{mbar}$ ) and a superconducting magnet.

Figure 1 clearly shows that $\mathrm{Co} / \mathrm{Pt}$ multilayers with outof-plane magnetization can exhibit perpendicular exchange bias when covered with or grown on top of an IrMn layer. In the as-deposited state the exchange-bias field is largest for

${ }^{a)}$ Electronic mail: vandijks@tcd.ie
$\mathrm{Co} / \mathrm{Pt}$ multilayers with IrMn on top. In this case, domain formation in the antiferromagnetic IrMn film is influenced by the out-of-plane moment on the Co surface. When the IrMn layer is grown first, no magnetic moment is present to establish a favorable domain structure in the antiferromagnet and this results in a smaller perpendicular exchange-bias field.

Annealing for $1 \mathrm{~h}$ at $220^{\circ} \mathrm{C}$ in an out-of-plane magnetic field of $5.5 \mathrm{~T}$ changes the exchange-bias field. For the top configuration the bias field decreases, whereas for the bottom configuration it increases. Since the magnetic history for both films is similar after the annealing process the difference in exchange-bias field is structural in origin. For IrMn exchange-biased systems with in-plane magnetization, a difference in the (111) film texture has been suggested as a possible explanation. ${ }^{2-7}$ However, XRD measurements on the annealed $\mathrm{Co} / \mathrm{Pt}$ multilayers only reveal a minor difference in (111) texture indicating that other structural parameters such as interface roughness and grain size have a

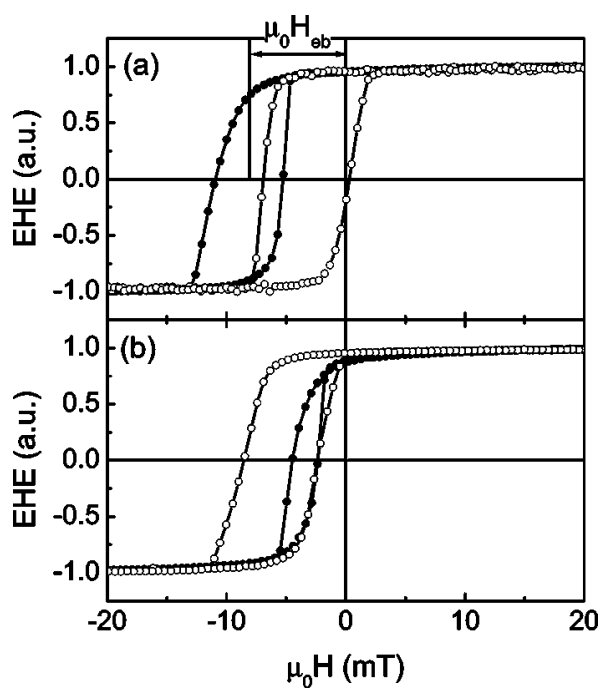

FIG. 1. Out-of-plane EHE hysteresis loops of a $\mathrm{SiO}_{2} /[\mathrm{Pt}(20 \AA) /$ Co $(4 \AA)]_{3} / \operatorname{IrMn}(100 \AA) / \mathrm{Pt}(20 \AA)$ multilayer (filled circles) and a $\mathrm{SiO}_{2} / \mathrm{Pt}(20 \AA) / \operatorname{IrMn}(100 \AA) /[\mathrm{Co}(4 \AA) / \mathrm{Pt}(20 \AA)]_{3}$ multilayer (open circles) before (a) and after (b) magnetic field annealing at $220{ }^{\circ} \mathrm{C}$. 


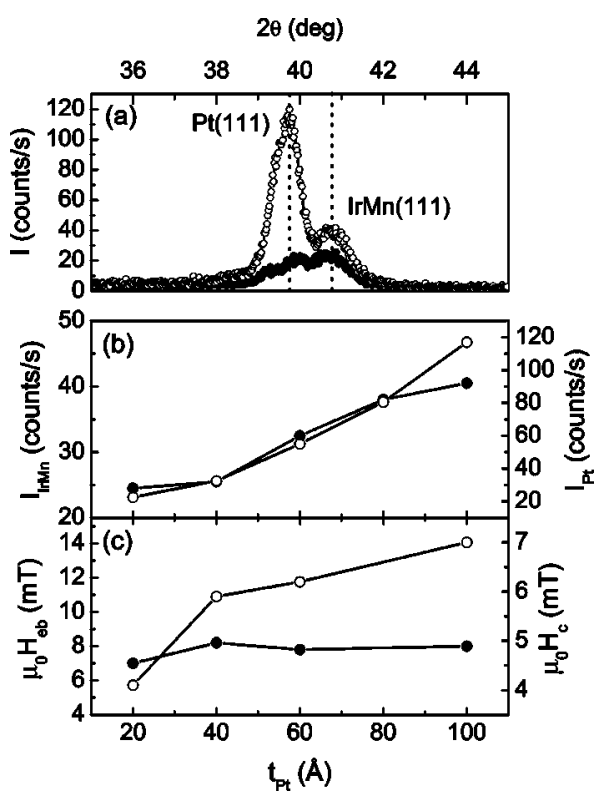

FIG. 2. (a) XRD data for $\mathrm{SiO}_{2} / \mathrm{Pt}(t \AA) /[\mathrm{Co}(5 \AA) / \mathrm{Pt}(20 \AA)]_{2} /$ Co $(5 \AA) / \operatorname{IrMn}(100 \AA) / \mathrm{Pt}(20 \AA)$ multilayers with $t=20 \AA$ (filled circles) and $t=100 \AA$ (open circles). (b) Intensity of the IrMn (111) reflection (filled circles) and Pt (111) reflection (open circles) as a function of Pt seed layer thickness. (c) Perpendicular exchange bias field (filled circles) and coercivity (open circles) as a function of Pt seed layer thickness.

greater influence on the exchange-bias interactions in the $\mathrm{Co} / \mathrm{Pt}-\mathrm{IrMn}$ system.

To study the influence of texture perfection on the perpendicular exchange-bias field in more detail, we grew a series of IrMn-covered $\mathrm{Co} / \mathrm{Pt}$ multilayers on different $\mathrm{Pt}$ seed layers. Figure 2(a) shows XRD scan for $\mathrm{SiO}_{2} /$ $\operatorname{Pt}(t \AA) /[\operatorname{Co}(5 \AA) / \operatorname{Pt}(20 \AA)]_{2} / \operatorname{Co}(5 \AA) / \operatorname{IrMn}(100 \AA) /$ Pt $(20 \AA)$ multilayers with $t_{\mathrm{Pt}}=20 \AA$ and $t_{\mathrm{Pt}}=100 \AA$ and Fig. 2(b) summarizes the dependence of the $\operatorname{IrMn}(111)$ and $\mathrm{Pt}(111)$ peak intensities on $t_{\mathrm{Pt}}$. As expected the XRD measurements reveal a clear enhancement of the (111) film texture with increasing Pt seed layer thickness. Although the more perfect initial Co/Pt growth on thicker Pt seed layers results in a well-known increase of the coercive field, ${ }^{20}$ the perpendicular exchange-bias field remains constant at about $8 \mathrm{mT}$ [see Fig. 2(c)]. From this we conclude that there is no direct relation between the degree of (111) texture and the perpendicular exchange-bias field in the $\mathrm{Co} / \mathrm{Pt}-\mathrm{IrMn}$ system.

The deposition of an IrMn film on top of a Co/Pt multilayer changes the magnetic anisotropy of the system. The magnetization of the multilayer remains out-of-plane if the effective magnetic anisotropy $K_{e f f}>0$. For Co/Pt multilayers $K_{\text {eff }}$ can be written as ${ }^{14,21}$

$$
K_{e f f}=K_{V}+\frac{(2 n-1) K_{S}^{\mathrm{Co} / \mathrm{Pt}}}{n t_{\mathrm{Co}}}+\frac{K_{S}^{t o p}}{n t_{\mathrm{Co}}},
$$

where $K_{V}$ is the volume anisotropy of the Co layers, which includes the shape and magnetocrystalline anisotropy, and $K_{S}^{\mathrm{Co} / \mathrm{Pt}}$ is the interfacial anisotropy of the $\mathrm{Co} / \mathrm{Pt}$ interface. For the unbiased and exchange-biased $\mathrm{Co} / \mathrm{Pt}$ multilayers, $K_{S}^{\text {top }}$ represents the interfacial anisotropy of the top Co/Pt interface $\left(K_{S}^{\mathrm{Co} / \mathrm{Pt}}\right)$ and the $\mathrm{Co} / \mathrm{IrMn}$ interface $\left(K_{S}^{\mathrm{Co} / \mathrm{IrMn}}\right)$, respec-

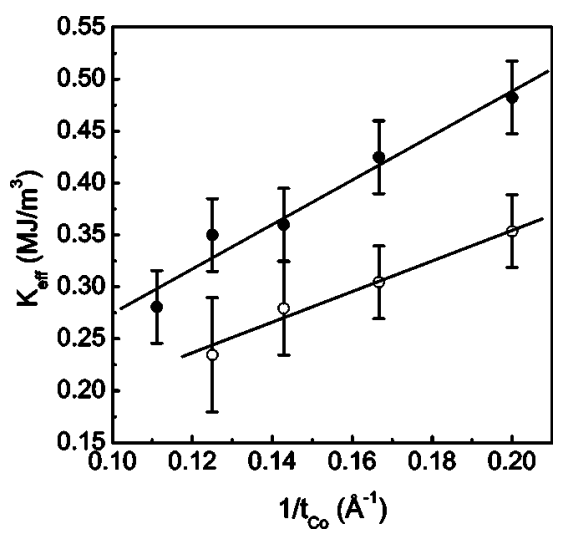

FIG. 3. Effective magnetic anisotropy as a function of the inverse Co layer thickness for $\mathrm{SiO}_{2} /[\mathrm{Pt}(20 \AA) / \mathrm{Co}(t \AA)]_{3} / \mathrm{Pt}(20 \AA)$ (filled circles) and a $\mathrm{SiO}_{2} /[\mathrm{Pt}(20 \AA) / \mathrm{Co}(t \AA)]_{3} / \operatorname{IrMn}(100 \AA) / \mathrm{Pt}(20 \AA) \quad$ (open circles) multilayers.

tively. We have estimated $K_{\text {eff }}$ from EHE measurements with an in-plane applied magnetic field. In these measurements the magnetization direction rotates coherently from perpendicular to parallel to the film plane. The anisotropy field $H_{k}$ necessary to obtain in-plane magnetization varies linearly with $K_{\text {eff }}$ and is approximately given by $H_{k}=2 K_{\text {eff }} / \mu_{0} M_{S}$. The resulting effective magnetic anisotropy of $\mathrm{Co} / \mathrm{Pt}$ multilayers with and without an IrMn top layer is shown as a function of $1 / t_{\mathrm{Co}}$ in Fig. 3. For the unbiased $\mathrm{Co} / \mathrm{Pt}$ multilayer the effective magnetic anisotropy increases with decreasing Co layer thickness and a fit to the data yields $K_{S}^{\mathrm{Co} / \mathrm{Pt}}=0.11 \mathrm{~mJ} / \mathrm{m}^{2}$. The effective anisotropy is smaller for the exchange-biased $\mathrm{Co} / \mathrm{Pt}$ multilayers. From the slopes of the two fits to the data in Fig. 3 the interfacial magnetic anisotropy of the $\mathrm{Co} / \mathrm{IrMn}$ interface can be determined. The result, $K_{S}^{\mathrm{Co} / \mathrm{IrMn}}=-0.09 \mathrm{~mJ} / \mathrm{m}^{2}$, indicates that the contribution of the $\mathrm{Co} / \mathrm{IrMn}$ interface to the effective magnetic anisotropy favours in-plane magnetization. Although the magnitude of this in-plane anisotropy is not enough to reorient the magnetization of the $\mathrm{Co} / \mathrm{Pt}$ multilayer from out-of-plane to in-plane, it might tilt the Co spins away from the film normal in the vicinity of the Co/IrMn interface. Such a misalignment between the magnetization of the top Co layer and the film normal will reduce the perpendicular exchange-bias field and this might be the main reason for the previously reported disappearance of exchange bias in Co/Pt-IrMn systems with a direct contact between the top Co layer and the IrMn film. ${ }^{19}$

It has recently been shown that the exchange bias of $\mathrm{Co} / \mathrm{Pt}$ multilayers with out-of-plane magnetization can be enhanced by the insertion of a thin Pt layer at the Co/AFM interface. ${ }^{15,19}$ Figure 4 shows that this also holds for our IrMn exchange-biased structures. For $\mathrm{SiO}_{2} /[\mathrm{Pt}(20 \AA) /$ Co $(5 \AA)]_{3} / \mathrm{Pt}(t \AA) / \operatorname{IrMn}(100 \AA) / \mathrm{Pt}(20 \AA)$ multilayers the perpendicular exchange bias initially increases and then decreases with the Pt insertion layer thickness. The dependence of $H_{e b}$ on $t_{\mathrm{Pt}}$ is a result of two competing effects: The increase of $H_{e b}$ is due to a reorientation of the Co spins towards the film normal $\left(K_{S}^{\mathrm{Co} / \mathrm{Pt}}\right.$ is positive $)$ and the decrease of $H_{e b}$ is a result of the exponential decay of the FM/AFM exchange bias interaction with nonmagnetic insertion layer 


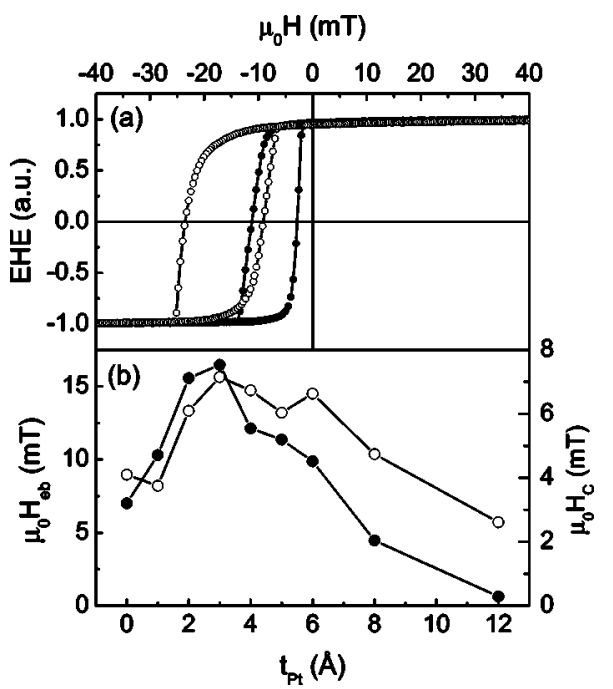

FIG. 4. Out-of-plane EHE hysteresis loops of $\mathrm{SiO}_{2} /[\mathrm{Pt}(20 \AA) /$ Co $(5 \AA)]_{3} / \mathrm{Pt}(t \AA) / \operatorname{IrMn}(100 \AA) / \mathrm{Pt}(20 \AA)$ multilayers with $t=0 \AA$ (filled circles) and $t=3 \AA$ (open circles). The data in (b) shows the dependence of the exchange bias field (filled circles) and the coercivity (open circles) on the thickness of the Pt insertion layer.

thickness. $^{22,23}$ The maximum exchange bias field is $16.5 \mathrm{mT}$ for $t_{\mathrm{Pt}}=3 \AA$, which is more than a factor 2 larger than that of the $\mathrm{Co} / \mathrm{Pt}$ multilayer without $\mathrm{Pt}$ insertion layer. The increase of $H_{e b}$ is accompanied by an enhancement of the coercive field.

In conclusion, we have shown that IrMn can be used to exchange-bias $\mathrm{Co} / \mathrm{Pt}$ multilayers with perpendicular magnetic anisotropy. For the perpendicular exchange-bias field we find that (1) in the as-deposited state $H_{e b}$ is largest for $\mathrm{Co} / \mathrm{Pt}$ multilayers with an IrMn film on top. (2) Magnetic field annealing enhances $H_{e b}$ for $\mathrm{Co} / \mathrm{Pt}$ multilayers with IrMn at the bottom. (3) $H_{e b}$ does not depend on the degree of (111) film texture. (4) $H_{e b}$ is limited by an in-plane magnetic anisotropy contribution from the $\mathrm{Co} / \mathrm{IrMn}$ interface $\left(K_{S}^{\mathrm{Co} / \mathrm{IrMn}}=-0.09 \mathrm{~mJ} / \mathrm{m}^{2}\right)$. (5) The insertion of a $3 \AA$ thick $\mathrm{Pt}$ layer at the $\mathrm{Co} / \mathrm{IrMn}$ interface more than doubles $H_{e b}$.

\section{ACKNOWLEDGMENT}

This work was supported by Science Foundation Ireland as part of the CINSE program.

${ }^{1}$ J. Nogués and I. K. Schuller, J. Magn. Magn. Mater. 192, 203 (1999).

${ }^{2}$ H. Fuke, K. Saito, Y. Kamiguchi, H. Iwasaki, and M. Sahashi, J. Appl. Phys. 81, 4004 (1997)

${ }^{3}$ A. J. Devasahayam, P. J. Sides, and M. Kryder, J. Appl. Phys. 83, 7216 (1998).

${ }^{4}$ J. van Driel, R. Coehoorn, K.-M. H. Lenssen, A. E. T. Kuiper, and F. R. de Boer, J. Appl. Phys. 85, 5522 (1999).

${ }^{5}$ J. van Driel, F. R. de Boer, K.-M. H. Lenssen, and R. Coehoorn, J. Appl. Phys. 88, 975 (2000).

${ }^{6}$ G. Anderson, Y. Huai, and L. Miloslawsky, J. Appl. Phys. 87, 6989 (2000).

${ }^{7}$ M. Pakala, Y. Huai, G. Anderson, and L. Miloslavsky, J. Appl. Phys. 87, 6653 (2000).

${ }^{8}$ S. Maat, K. Takano, S. S. P. Parkin, and E. E. Fullerton, Phys. Rev. Lett. 87, 087202 (2001)

${ }^{9}$ O. Hellwig, S. Maat, J. B. Kortright, and E. E. Fullerton, Phys. Rev. B 65, 144418 (2002).

${ }^{10}$ T. L. Kirk, O. Hellwig, and E. E. Fullerton, Phys. Rev. B 65, 224426 (2002).

${ }^{11}$ P. Kappenberger, S. Martin, Y. Pellmont, H. J. Hug, J. B. Kortright, O. Hellwig, and E. E. Fullerton, Phys. Rev. Lett. 91, 267202 (2003).

${ }^{12}$ Z. Y. Liu and S. Adenwalla, J. Appl. Phys. 94, 1105 (2003).

${ }^{13}$ F. Garcia, G. Casali, S. Auffret, B. Rodmacq, and B. Dieny, J. Appl. Phys. 91, 6905 (2002).

${ }^{14}$ F. Garcia, F. Fettar, S. Auffret, B. Rodmacq, and B. Dieny, J. Appl. Phys. 93, 8397 (2003).

${ }^{15}$ F. Garcia, J. Sort, B. Rodmacq, S. Auffret, and B. Dieny, Appl. Phys. Lett. 83, 3537 (2003).

${ }^{16}$ C. H. Marrows, Phys. Rev. B 68, 012405 (2003).

${ }^{17}$ L. Sun, S. M. Zhou, P. C. Searson, and C. L. Chien, J. Appl. Phys. 93, 6841 (2003)

${ }^{18}$ S. M. Zhou, L. Sun, P. C. Searson, and C. L. Chien, Phys. Rev. B 69, 024408 (2004).

${ }^{19}$ J. Sort, F. Garcia, B. Rodmacq, S. Auffret, and B. Dieny, J. Magn. Magn. Mater. 272-276, 355 (2004)

${ }^{20}$ W. B. Zeper, H. W. van Kesteren, B. A. J. Jacobs, J. H. M. Spruit, and P. F. Carcia, J. Appl. Phys. 70, 2264 (1991).

${ }^{21}$ F. J. A. den Broeder, H. C. Donkersloot, H. J. G. Draaisma, and W. J. M. de Jonge, J. Appl. Phys. 61, 4317 (1985).

${ }^{22}$ N. J. Gökemeijer, T. Ambrose, and C. L. Chien, Phys. Rev. Lett. 79, 4270 (1997).

${ }^{23}$ L. Thomas, A. J. Kellock, and S. S. P. Parkin, J. Appl. Phys. 87, 5061 (2000). 